Wolters, G. F.

Kluyver, J.C.

1955

\title{
INFLUENCE OF THE DISTANCE BETWEEN SOURCE AND CRYSTAL ON THE DETECTION-EFFICIENCY OF A GAMMA- SCINTILLATION SPECTROMETER
}

\author{
by L. H. TH. RIETJENS, G. J. ARKENBOUT, G. F. WOLTERS and \\ J. C. KLUYVER
}

Physisch Lahoratoriun der Rijksuniversiteit te litrerht, Nederland

\section{Synopsis}

The influence of the distance between source and crystal on the counting efficiency of a scintillation spectrometer has been studied. For gamma-rays of energy larger than $400 \mathrm{keV}$ the efficiency may vary by a factor of two depending on the distance from source to crystal. The experimentally observed dependence may be fully explained by simple geometrical arguments.

$\S 1$. Introduction. The measurement of $\gamma$-ray energies by means of a scintillation spectrometer has now become standard procedure. By careful calibration a precision of $1 \%$ or better may be obtained. Intensity measurements with scintillation spectrometers, however, seldom reach an accuracy better than $15 \%$. For $\gamma$-ray energies up to $1.5 \mathrm{MeV}$ the total number of counts in the full-energy peak (photopeak) is the obvious measure for the intensity. Thus the photopeak efficiency will be defined as the number of counts in the photopeak divided by the number of $\gamma$-rays incident on the surface of the crystal. The full-energy peak is not only formed by the $\gamma$-rays which yield a single photo-effect, but also by those $\gamma$-rays of which the total energy is absorbed in the crystal by multiple processes, e.g. a Compton scattering followed by photo-effect of the scattered quantum. The relative area under this peak, however, depends not only, as is well known, on the energy of the $\gamma$-ray and the dimensions of the crystal, but also on the distance between source and crystal. The origin of this effect is due to the fact, that the average length of the path in the crystal depends on the direction of the incident $\gamma$-ray.

Attention to this effect has already been drawn by $\mathrm{Ste} \mathrm{rk}$ and $\mathrm{W}$ a $\mathrm{p}-$ st $\mathrm{ra}^{1}$ ), and $\mathrm{H}$ ornyak and $\mathrm{Coor}{ }^{2}$ ), but no attempt has been made to confront the experimental results with geometrical considerations.

In the present investigation the photopeak efficiencies for several $\gamma$-rays have been measured for distances between 3 and $90 \mathrm{~mm}$ from source to crystal. As will be shown (Fig. 4) the ratio of the photopeak efficiencies for distances of 7 and $90 \mathrm{~mm}$ from source to crystal is of the order of 0.55 for $\gamma$-ray energies larger than $400 \mathrm{keV}$. It is evident that effects of this magnitude are of importance in accurate intensity measurements. 
The dependence of the efficiency on the distance from source to crystal has also been calculated. When equation I ( $\$ 3$ ) is applied, complete agreement between experiment and calculations is obtained. It thus seems justified to use the theory also for crystals of other dimensions than those of the present experiment.

For $\gamma$-rays up to $E_{\gamma}=150 \mathrm{keV}$ the efficiency calibration of $\gamma$-scintillation spectrometers is best done by absorption measurements. For $\gamma$-rays of energy $>150 \mathrm{keV}$ use is made of sources emitting several $\gamma$-rays and X-rays of known intensity ratio. The treatment of the source-crystal distance effect has accordingly been divided up into two parts ( 33 and $\S 4)$.

$\S 2$. Experimental equipment. All $\gamma$-ray measurements have been made with a $\gamma$-scintillation spectrometer, consisting of a $\mathrm{NaI}(\mathrm{Tl})$ crystal (in the standard Harshaw mounting), a Du Mont 6292 photo-multiplier tube, a linear amplifier and a one-channel differential discriminator. The crystal is a circular cylinder and has a diameter and a length of $25.4 \mathrm{~mm}$. The photopeak halfwidth is $10 \%$ at $E_{y}=511 \mathrm{keV}$ (annihilation radiation).

The isotopes: ${ }^{22} \mathrm{Na},{ }^{88} \mathrm{Y},{ }^{137} \mathrm{Cs}$ and ${ }^{203} \mathrm{Hg}$ were used for the calibrations. In all experiments the diameter of the source was smaller than $5 \mathrm{~mm}$. The thickness was always such, that no correction for self-absorption had to be applied, and was in all cases less than $1 \mathrm{~mm}$.

When necessary, corrections have been applied for the absorption in the $\mathrm{Al}$-cover and the $\mathrm{MgO}$-reflector of the crystal. These corrections are again dependent on the distance between source and crystal, because oblique incidence has to be taken into account.

§3. Efficiencies by absorption measurements. For $\gamma$-radiation of energy $E_{\gamma}<150 \mathrm{keV}$ each $\gamma$-quantum absorbed by the crystal contributes to the photopeak, if the escapepeak is included $\left.{ }^{3}\right){ }^{4}$ ). In this region the photoabsorption coefficient is equal to the total absorption coefficient, which is determined by measuring the absorption in a $\mathrm{NaI}(\mathrm{Tl})$ slice of $1.20 \times 30 \times 30$ $\mathrm{mm}^{3}$. The distance between the source and crystal was $90 \mathrm{~mm}$, so that one may assume that all $\gamma$-rays pass through the slice at right angles of incidence.

From the measured photo-absorption coefficients, the photopeak efficiency of the crystal can be computed numerically.

Introducing the following notation:

$\tau=$ photo-absorption coefficient,

$\alpha=$ angle between the axis of the crystal and the direction of the emitted $\gamma$-quantum (for $\alpha_{0}$ and $\alpha_{1}$ see Fig. 1),

$l(\alpha)=$ path through the crystal of the $\gamma$-rays making an angle $\alpha$ with the axis if there were no absorption,

$D=$ distance between the source and the front surface of the crystal,

$l=$ length of the crystal,

$r=$ radius of the front surface of the crystal, 
the photo-efficiency $(\varepsilon)$ for a cylindrical crystal is given by:

$$
\begin{aligned}
& \varepsilon=1-\frac{\int_{0}^{\alpha_{0}} \frac{1}{2} \sin \alpha \cdot \exp \{-\tau l(\alpha)\} \mathrm{d} \alpha}{\int_{0}^{a_{0}} \frac{1}{2} \sin \alpha \mathrm{d} \alpha}= \\
& =1-\frac{\int_{0}^{a_{1}} \sin \alpha \cdot \exp \left(\frac{-\tau l}{\cos \alpha}\right) \mathrm{d} \alpha+\int_{a_{1}}^{a_{0}} \sin \alpha \cdot \exp \left\{-\tau\left(\frac{r}{\sin \alpha}-\frac{D}{\cos \alpha}\right)\right\} \mathrm{d} \alpha}{1-\cos \alpha_{0}}
\end{aligned}
$$

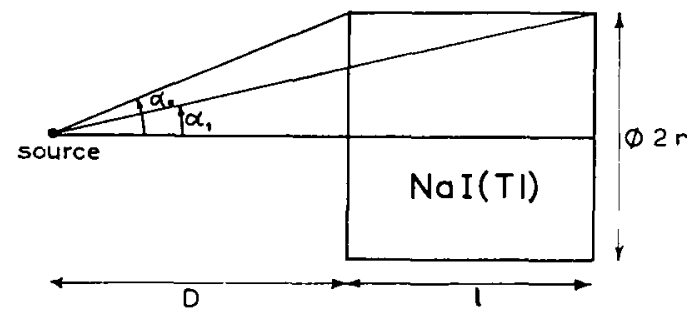

Fig. 1. Geometry source-crystal. The crystal is of cylindrical form with $l=2 r=25.4 \mathrm{~mm}$.

The integral has been divided up into two parts. The integral between $a=0$ and $\alpha=\alpha_{1}$ can be easily evaluated, for in this region $l(\alpha)$ can be approximated (within $1 \%$ ) by:

$$
\bar{l}\left(\alpha_{1}\right)=\frac{\int_{0}^{a_{1}} \frac{1}{2} \sin \alpha l(\alpha) \mathrm{d} \alpha}{\int_{0}^{a_{1}} \frac{1}{2} \sin \alpha \mathrm{d} \alpha}=\frac{-l \ln \cos \alpha_{1}}{1-\cos \alpha_{1}}
$$

The integral between $\alpha=\alpha_{1}$ and $\alpha=\alpha_{0}$ has to be computed numerically.

The results are given in Table I (column 4 and 5), together with the measured absorption coefficients (column 3 ).

TABLE I

\begin{tabular}{|c|c|c|c|c|}
\hline \multirow{2}{*}{ Isotope } & \multirow{2}{*}{$E_{\gamma}(\mathrm{keV})$} & $\tau\left(\mathrm{mm}^{-1}\right)$ & \multicolumn{2}{|c|}{ photopeak efficiency } \\
\hline${ }^{137} \mathrm{Cs}$ & 31 & $2.17 \pm 0.15$ & 0.995 & 0.98 \\
$203 \mathrm{Hg}$ & 70 & $1.48 \pm 0.10$ & 0.99 & 0.96 \\
& 102 & $\left.0.55^{*}\right)$ & 0.97 & 0.91 \\
& 153 & $\left.0.172^{*}\right)$ & 0.89 & 0.72 \\
\hline
\end{tabular}

*) Dr. D. Ma eder (E.T.H., Zürich) kindly informed us of his data at these energies.

As in this energy region $\left(E_{\gamma}<150 \mathrm{keV}\right)$ a very thin layer of $\mathrm{NaI}$ suffices for absorption of all incident $\gamma$-rays, the source-crystal distance and thus the angle of incidence of the $\gamma$-rays, is not very important to the detection efficiency of the crystal. Nevertheless, already for $E_{\gamma}>100 \mathrm{keV}$ the dependence of the efficiency on $D$ may no longer be neglected.

$\S 4$. Efficiencies for $\gamma$-radiation with $E_{\gamma}$ larger than $150 \mathrm{keV}$. In the region of $E_{\gamma}>150 \mathrm{keV}$ not all $\gamma$-quanta absorbed by the crystal contribute to the photopeak. Here the efficiency of the crystal can be determined using sources 
emitting several $\gamma$-rays and $\mathrm{X}$-rays of known intensity ratio. The efflciency of the higher energy $\gamma$-ray is ${ }^{5}{ }^{6}$ ):

$$
\varepsilon_{2}=\varkappa \cdot \varepsilon_{1} \cdot S_{2} / S_{1}
$$

Where $\varepsilon_{1}=$ known efficiency of the X-rays (see $\$ 3$ ) or the efficiency of the lower-energy $\gamma$-ray obtained by interpolation,

$S_{1}$ and $S_{2}=$ areas of the photopeaks,

$x=$ intensity ratio of the X-and $\gamma$-ray or of the two $\gamma$-rays.

For two distances between source and crystal viz. 7 and $90 \mathrm{~mm}$ the ratio of the areas under the photopeaks $\left(S_{2} / S_{1}\right)$ was determined from the scintillation spectra. The results together with the corresponding efficiencies $\varepsilon_{2}$ are collected in Table II and plotted in Fig. 2 and show clearly the influence of the distance between source and crystal on the photopeak efficiency.

TABLE II

\begin{tabular}{|c|c|c|c|c|c|c|c|c|c|c|}
\hline \multirow[b]{2}{*}{ Isotope } & \multirow{2}{*}{$E_{1}(k e V)$} & \multirow{2}{*}{$E_{2}(\mathrm{keV})$} & \multirow[b]{2}{*}{$x$} & \multicolumn{2}{|c|}{$S_{2} / S_{1}$} & \multicolumn{2}{|c|}{$\begin{array}{c}\text { Experimental } \\
\varepsilon_{2}\end{array}$} & \multirow{2}{*}{$\begin{array}{c}\tau_{E_{2}}\left(\mathrm{~mm}^{-1}\right) \\
\text { full } \\
\text { energy } \\
\text { peak }\end{array}$} & \multicolumn{2}{|c|}{$\begin{array}{c}\text { Calculated } \\
\varepsilon_{\mathbf{a}}\end{array}$} \\
\hline & & & & $\begin{array}{c}D= \\
90 \mathrm{~mm}\end{array}$ & $\begin{array}{c}D= \\
7 \mathrm{~mm}\end{array}$ & $\begin{array}{c}\mathrm{D}= \\
90 \mathrm{~mm}\end{array}$ & $\begin{array}{l}D= \\
7 \mathrm{~mm}\end{array}$ & & $\begin{array}{c}D= \\
90 \mathrm{~mm}\end{array}$ & $\begin{array}{c}D= \\
7 \mathrm{~mm}\end{array}$ \\
\hline${ }^{203} \mathrm{Hg}$ & 70 & 279 & $\left.0.146^{5}\right)$ & 2.71 & 1.81 & 0.391 & 0.253 & 0.0265 & 0.395 & 0.256 \\
\hline${ }^{137} \mathrm{Cs}$ & 31 & 662 & $\left.0.081^{\circ}\right)$ & 1.34 & 0.85 & 0.109 & 0.067 & 0.0059 & 0.109 & 0.063 \\
\hline${ }^{22} \mathrm{Na}$ & 511 & 1277 & $\left.1.78^{\prime}\right)$ & 0.141 & 0.135 & 0.042 & 0.023 & 0.00222 & 0.042 & 0.022 \\
\hline${ }^{89} \mathrm{Y}$ & 910 & 1850 & 1 & - & 0.36 & - & 0.015 & - & - & - \\
\hline
\end{tabular}

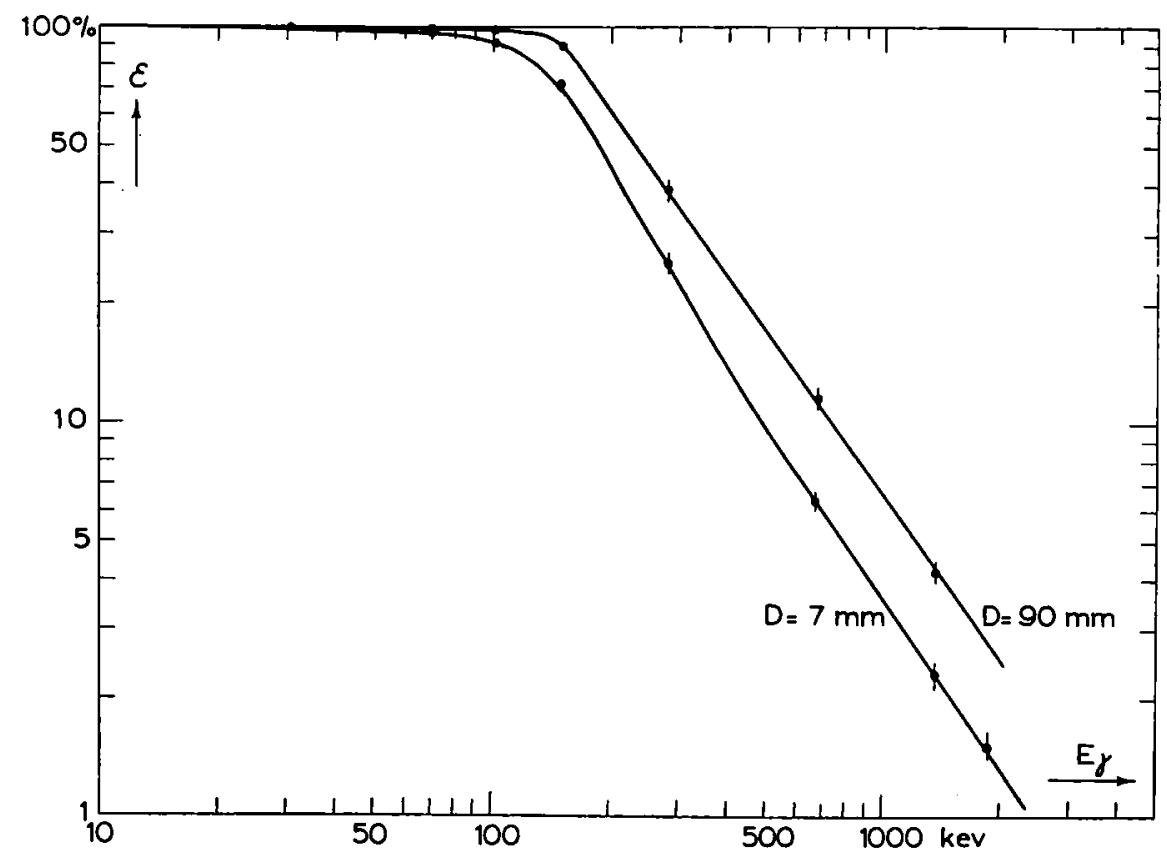

Fig. 2. The efficiency curves for a cylindrical crystal with a cliameter and a length of $25.4 \mathrm{~mm}$, for distances of $90 \mathrm{~mm}$ and $7 \mathrm{~mm}$ between source and crystal. 
The dependence of the photopeak efficiency on the distance has been given in Eq. I. The $\tau$ from Eq. I does not only refer to the number of photoeffects, but also to all multiple processes, which contribute to the full-energy

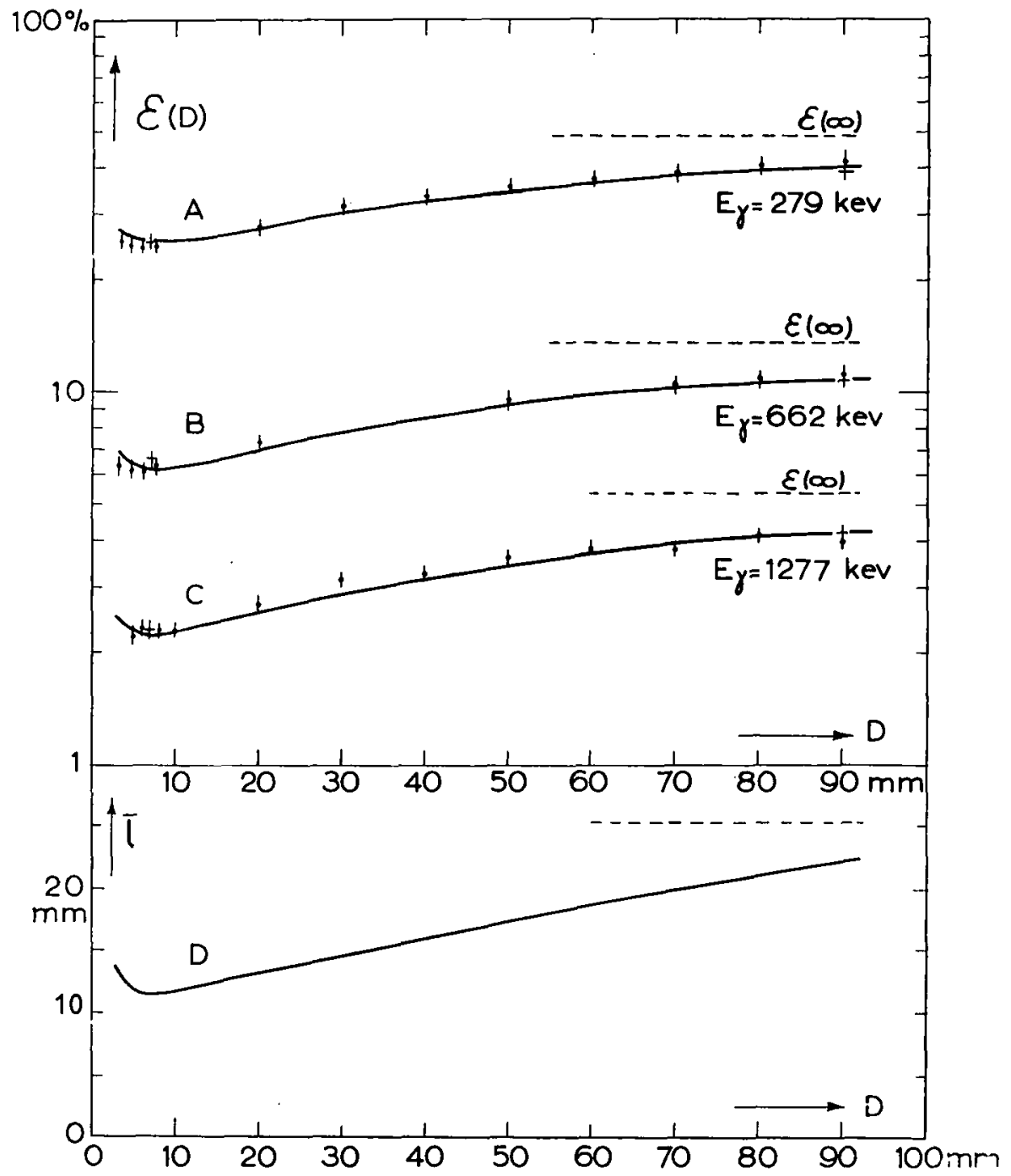

Fig. 3. The efficiency as a function of the distance between source and crystal. The curves $\mathrm{A}, \mathrm{B}$ and $\mathrm{C}$ are calculated for $\gamma$-radiation with $E_{\gamma}=279 \mathrm{keV}, 662 \mathrm{keV}$ and $1277 \mathrm{keV}$. The crosses and dots are experimental points obtained by using the method given by Eq. III respectively Eq. IV. Curve $D$ represents the mean $\gamma$-ray path through the crystal for the hypothetical case of no absorption.

peak, and for $\mathrm{E}_{\gamma}>150 \mathrm{keV}$ this second contribution is no longer negligible.

In this case $\tau$ has to be determined from the measured efficiency by using Eq. I. But as $\tau$ cannot be expressed explicitly in $\varepsilon$, first the approximation 
$\tau_{1}=-\bar{l}^{-1} \ln (1-\varepsilon)$ is used where $\bar{l}$ is the mean $\gamma$-ray path (cf. Fig. 3D) through the crystal. This $\tau_{1}$ and a slightly different value $\tau_{2}$ are substituted in Eq. I. The so obtained efficiencies $\varepsilon_{1}$ and $\varepsilon_{2}$ will differ from the experimental efficiency. The correct value of $\tau$ may then be found by interpolation. So it has been tried to find for each energy one $\tau$ that would fit equation I both for distances $D=90 \mathrm{~mm}$ and $D=7 \mathrm{~mm}$. In the last three columns of Table II the corresponding $\tau$ is given, with the ensuing efficiencies. The fact, that these calculated efficiencies agree with the experimental values proves the adequacy of Eq. I.Thus with this value of $\tau$ the efficiency for any distance may be computed. The results of these calculations are plotted in Fig. 3 for the $279 \mathrm{keV} \gamma$-ray of ${ }^{203} \mathrm{Hg}$, for the $662 \mathrm{keV} \gamma$-ray of ${ }^{137} \mathrm{Cs}$ and for the $1277 \mathrm{keV}$ $\gamma$-ray of ${ }^{22} \mathrm{Na}$. The experimental data have been obtained in two ways:

1) In the first place data for distances of 90 and $7 \mathrm{~mm}$ have been obtained by the method mentioned in $\S 4$ (Eq. III). See also Table II.

2) Further data have been obtained by using:

$$
\varepsilon(D)=\frac{N_{p h}(D)}{N_{0} \cdot \Omega(D)}
$$

where $\varepsilon(D)=$ efficiency dependent on the distance $D$,

$N_{p h}(D)=$ number of pulses in the photopeak per sec,

$N_{0}=$ total number of $\gamma$-quanta emitted per sec,

$4 \pi \Omega(D)=$ solid angle from source to crystal $=2 \pi\left(1-\cos \alpha_{0}\right)$, and $N_{0}$ has been adjusted to fit the curve.

$\S 5$. Discussion. Both Fig. 2 and 3 demonstrate clearly the influence of the distance $D$ on the photopeak efficiency. The experimental effects are, within statistical errors, in very good agreement with the calculations based on geometrical considerations. This agreement is once more demonstrated in Fig. 4. In this figure the ratio of efficiencies at distances $D=7 \mathrm{~mm}$ and $D=90 \mathrm{~mm}$ is plotted as a function of the energy $\left(E_{\gamma}\right)$. If the photo-efficiency were independent of the distance this ratio would be equal to 1 for all energies. The curve in Fig. 4 represents the calculated curve based on geometrical considerations.

Moreover, the good agreement between experiments and calculations confirms the assumption that the absorption coefficient is independent of the distance $D$ between source and crystal. As also multiple processes contribute to $\tau$, and the probability of multiple processes certainly depends on the place in the crystal where the first Compton effect occurs, this assumption cannot hold rigidly, but is a good enough approximation to enable the calculation of the variation of the efficiency with the distance $D$ to be carried out with an accuracy of ca. $7 \%$. This is about the same precision as can be obtained in direct measurements. The uncertainty in the determination of $S_{2} / S_{1}$ and in the value used for the intensity ratio $\varkappa$ (cf. Eq. III) impose this limit. 
As the intensities of the radioactive sources to be studied may vary by several factors of 10 , it will often be convenient to place various sources at different distances from the crystal. In this case it is necessary to know the photopeak efficiency of the crystal for all energies as a function of the sourcecrystal distance. With the results of this paper it will be possible to calibrate

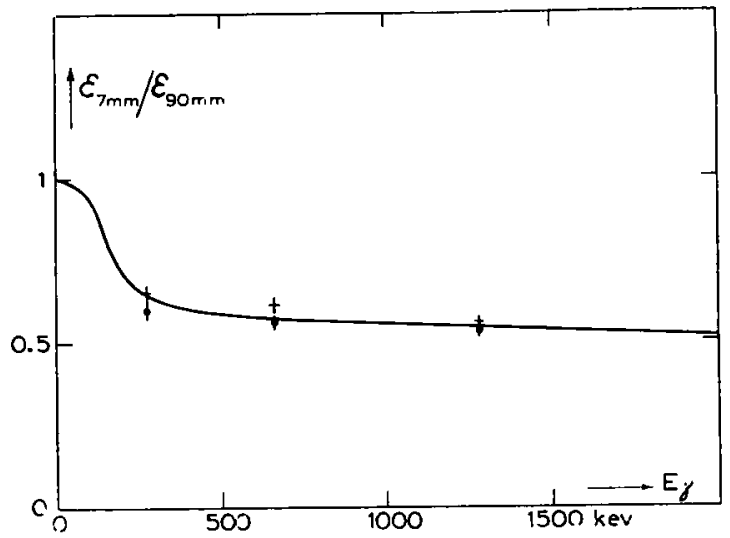

Fig. 4. The ratio of the efficiencies at distances of 7 and $90 \mathrm{~mm}$ as a function of the $\gamma$-ray energy. The drawn curve is based on calculations. The crosses and dots are the experimental points obtained by using Eq. III respectively Eq. IV.

a scintillation spectrometer with a crystal of arbitrary dimensions in the following way: the efficiencies for several $\gamma$-ray energies are measured at one source-crystal distance only, the absorption coefficients $\tau$ for this crystal are calculated from Eq. I and the efficiencies for other distances are again obtained with the aid of Eq. I.

Acknowledgements. This investigation is part of the research program of the group Utrecht (under the direction of Professor J. M. W. M i la t z) of the "Stichting voor Fundamenteel Onderzoek der Materie" and was made possible by a subvention from the "Nederlandse Organisatie voor Zuiver Wetenschappelijk Onderzoek". The authors are indebted to Professor P. M. End $\mathrm{t}$ and Dr. H. J. van de $\mathrm{n} \mathrm{Bold}$ for valuable discussions and comments.

Recejved 19-11-54.

\section{REFERENCES}

1) Sterk, M. J. and $W$ a p stra, A. H., Physica 19 (1953) 669.

2) Horn y a k, W. F. and C o or, T., Phys. Rev. 92 (1953) 675.

3) Nove y, T. B., Phys. Rev. 89 (1953) 672.

4) A x e 1, P., Brookhaven National Laboratory 271 (T-44) (1953).

5) Wapstra, A. H., Thesis Amsterdam (1953) 28.

6) Aström, Wa pstra, Thu lin and Bergström, Arkiv Fysik 7 (1953) 247.

7) Sherr, R. and Miller, R. H., Phys. Rev. 93 (1954) 1076.

8) Hollander, Perlman, Sc a borg, Rev. mod. Phys. 25 (1953) 517. 